\title{
Beyond The Extreme Abuse Survey: Exploring Technological, Social, Non-physical, Bio-energetic and Criminological Aspects
}

\section{Summary}

The set of Extreme Abuse Surveys (EAS) of 2007 produced a detailed and coherent profile of systematic trauma procedures. This poster presents samples of additional types of experiences reported by survivors of organised (child) abuse based on Howard (2018). It explores reoccurring themes that witnesses report having lived through, and aims to promote further research into the energetic, social, criminological, and technological aspects of mind control. This gives arguments and motive for clarifying the impact of this type of offending on mental health, as well as for development of deeper insights in therapeutic practice that counter vested interests.
Dr Rainer Hermann Kurz

C.Psychologist

ichinendaimoku@gmail.com

Mr Declan Howard

Expert by Experience

fieldmouse@gmx.es

\section{Background}

In the midst of the 'Memory Wars' of the 1990s a survey by the BPS Working Group on recovered memory revealed that $15 \%$ of clinical practitioners had encountered client reports of Satanist Ritual Abuse (SRA) but failed to describe or define it.

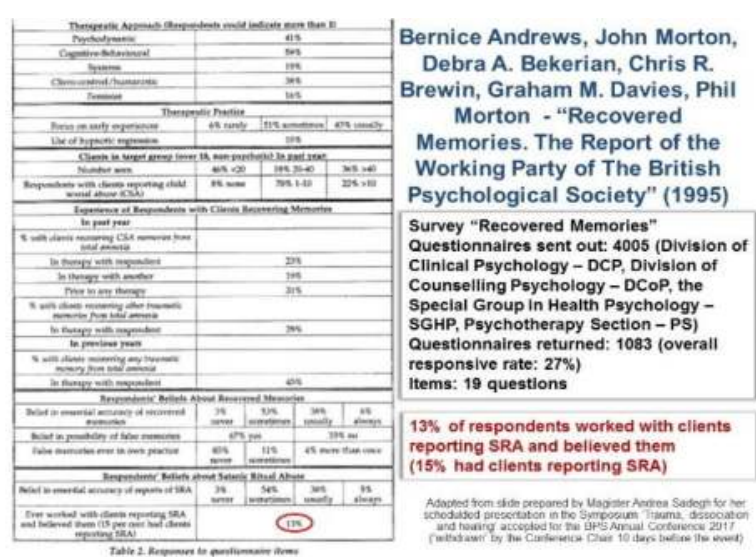

Kurz (2017) slide illustrating nature and key results of the BPS Survey on Traumatic Memories

The Extreme Abuse Survey (Becker, Karriker, Overkamp, \& Rutz, 2008) covered numerous extreme offences and complex trauma symptoms summarised by Kurz (2018). Kurz \& Howard (2018) reported on a self-healing approach for recovery from such abuse. Having identified gaps in the EAS coverage, Howard (2018) issued a manuscript that attempts to include features that were not surveyed at that time, going beyond the territory defined by materialist scientific orthodoxy. The wide array of questions is based on the testimony of multiple survivors and raises fundamental questions. Method

In order to present a modus operandi of abuse syndicates, disclosures of traumatic memories across a range of witnesses were pooled, categorised and presented in the format of survey prompts.

Results

The first (A) section explores the use of technology including electroshock, simulations and directed energy weapons. Aaron (2017) provides an excellent overview of the latter explaining how deniability is central to their deployment. The second (B) set of questions includes genetics, families and coerced breeding. The third (C) features energy body and psyche themes referring to programming and extended faculties. The fourth (D) covers entities and other external non-physical situations. The fifth (E) explores the obstacles to healing, communication and inclusion found in society. The last section $(F)$ features crimes such as organised abuse, animal sacrifice, murder and forced perpetration.

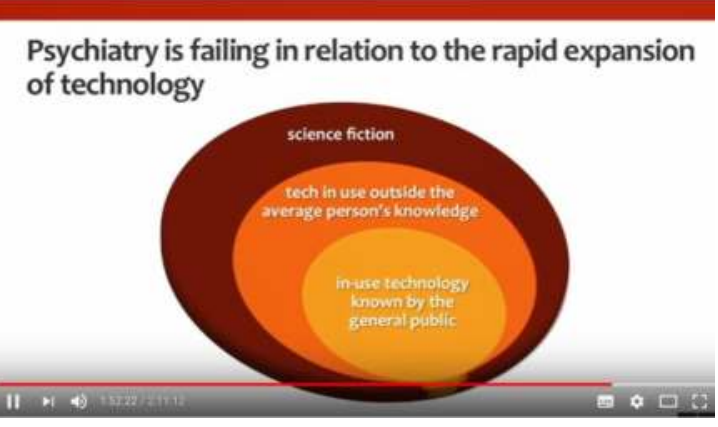

Slide in presentation of Matthew Aaron (2017) illustrating the pivotal role of Psychiatry

https://www.youtube.com/watch?v=WdOWi_IJ-dU

Illustrative sample questions encountered...')

A1. The use of directed energy weapons?

A15. Devices implanted in the body?

A17. Experiences that took place in a simulator?

A18. Alien abductions or mock alien abductions?

\section{B6. Being cloned?}

B15. Have you witnessed shapeshifting to a reptilian? B23. Being impregnated to produce a baby for the cult? B26. Have you had a baby who was taken away?

C1. Etheric implants in the energy body?

C15. Traumatic procedures which deformed and damaged the energy body?

C22. Telepathy, remote viewing or precognition? C23. Mother-child bond broken or damaged?

D1. Attachment to energetic parasites, e.g. demons? D4. Being influenced or controlled by external entities?

D20. Psychic battles?

D21. Receiving messages, guidance, or instructions from non-physical sources?

\section{E1. Gaslighting?}

E8. Difficulty communicating due to public ignorance of child trafficking?

E23. Trying to warn people but being disregarded, ignored, belittled or mocked?

E24. Being directed to psychological and psychiatric services instead of law enforcement?

F7. Abused by a politician, celebrity, aristocrat, or person in position of authority?

F16. Abuse in the womb?

F35. The drinking of blood?

F56. Made to kill another child by being physically manipulated?
Aspects of extreme and systematic trauma reported by survivors - view / download essay / survey: https://lonehorseblog.files.wordpress.com/2018/01/aspects-1.pdf

http://endritualabuse.org/findings-from-the-2007-extreme-abuse-survey-eas-series/ https://extreme-abuse-survey.org/survey.php?en=b

https://www.telegraph.co.uk/science/2018/03/22/bizarre-six-inch-alien-skeleton-found-buried-desert-human-baby/

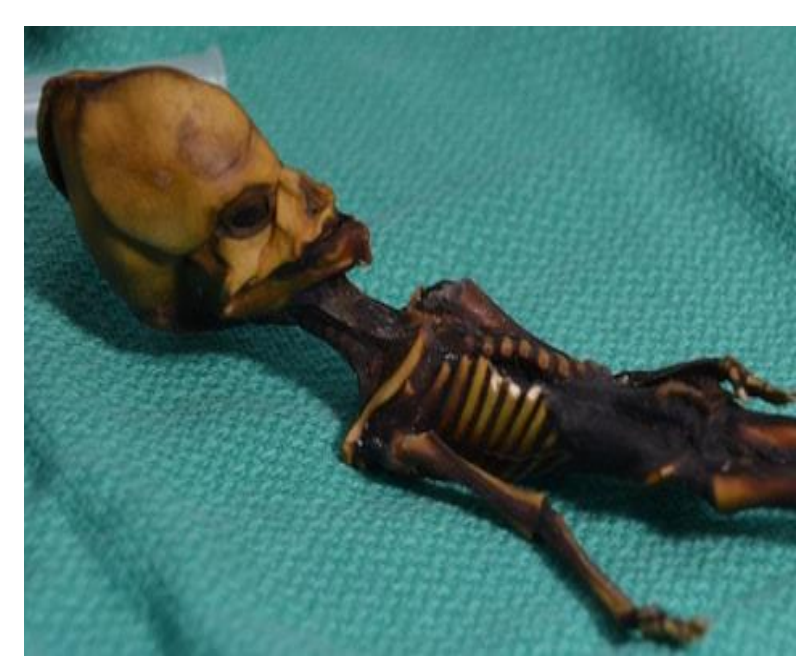

'A bizarre six-inch skeleton was discovered buried in a leather pouch behind an abandoned church in the Atacama Desert of Chile in 2003.

\section{Discussion}

The manuscript is published as a resource to guide investigators who are trying to profile and stop these crimes, and for psychotherapists, psychologists and psychiatrists so they can equip themselves to meet the needs of their clients.

In medieval times Galileo Galilei was forced to recant his belief that the earth is round and circulates around the sun. If a 'bizarre six-inch skeleton' that looks like an alien can be discussed in mainstream media why is it such a taboo to explore experiences concerned with non-human life forms?

Failure to engage with the disclosures of trauma victims is unscientific and facilitates the cover-up of offences. Witness testimonies indicate trauma based mind control criminality to be more developed than first thought in scale, organisation, technology and degeneracy. Psychiatrists and psychologists have a duty to 'step up to the plate' and consider extreme abuse disclosures with due care.

\section{References}

Aaron, Matthew (2017). No-Touch Microwave Torture by Electronic Harassment - New Socio-Criminal Problem of Global Proportions. 1st Annual Unity \& Hope Conference for Targeted Individuals in Boston, MA, Oct. 21, 2017.

Andrews, B., Morton, J., Bekerian, D. A., Brewin, C. R., Davies, G. M. \& Morton, P. (1995). The recovery of memories in clinical practice. Experiences and beliefs of British Psychological Society practitioners. In: The Psychologist. BPS: Leicester.

Becker, T., Karriker, W., Overkamp, B. \& Rutz, C. (2008). The extreme abuse surveys: preliminary findings regarding dissociative identity disorder. In Sachs, A. \& Galton, G. (Eds.): Forensic aspects of dissociative identity disorder. Karnac Books: London.

Kurz, R. (2018). BPS WORKING GROUP ON RECOVERED MEMORIES \& EXTREME ABUSE SURVEYS. Poster at the EPA Congress in Nice.

Kurz, R. (2018). TRAUMA-BASED MIND CONTROL \& ISTANBUL PROTOCOL FOR TORTURE DIAGNOSIS. Poster at the EPA Congress in Nice.

Kurz, R. (2018). BPS WORKING GROUP ON RECOVERED MEMORIES \& EXTREME ABUSE SURVEYS. Poster at the EPA Congress in Nice. 